\title{
The metastable states in submicron elliptical thin films
}

\author{
Mei-Feng Lai ${ }^{\mathrm{a}}$, Zung-Hang Wei ${ }^{\mathrm{a}, *}$, Ching-Ray Chang ${ }^{\mathrm{a}}$, N.A. Usov ${ }^{\mathrm{b}}$, J.C. Wu ${ }^{\mathrm{c}}$, \\ Jun-Yang Lai ${ }^{\mathrm{a}}$ \\ a Department of Physics, National Taiwan University, Taipei 10617, Taiwan \\ ${ }^{\mathrm{b}}$ Troitsk Institute for Innovation and Fusion Research, Troitsk, Moscow Region 142190, Russia \\ ${ }^{\mathrm{c}}$ Department of Physics, National Changhua University of Education, Changhua 500, Taiwan
}

\begin{abstract}
We investigate numerically the magnetization structures of the metastable states including quasiuniform state and various vortex states existing in an elliptical permalloy thin film. The typical energy curves as functions of the particle's size are obtained. It is found that the energy density of the quasiuniform state is smaller than the energy densities of these vortex states for small particles, but after a narrow transition range of size the energy density of the quasiuniform state becomes larger than the energy densities of these vortex states.
\end{abstract}

(C) 2004 Elsevier B.V. All rights reserved.

PACS: $75.60 . \mathrm{Ch} ; 75.75 .+\mathrm{a}$

Keywords: Quasiuniform state; Vortex state; Micromagnetic simulation

For nano-structured particles, the single-domain state is the most stable state, while for submicron-sized particles the single-domain state is not the only stable state at remanence. Instead, various metastable states energetically compete with the single-domain state. However, the observed states depend on the particle's dimension, shape, material properties, magnetization history [1], and edge roughness.

Studies have been carried out for the magnetization configurations of rectangles [2]. In ferromagnetic particles with sharp corners, such as rectangular thin films, the edge domains induced by sharp corners might cause some nucleation centers, which influence the stable states and reversal behaviors. In an elliptical particle, however, because of the absence of sharp corners the quasiuniform state is similar to the single-domain state of ellipsoidal particle, which has uniform magnetization configuration. For simplification and potential application, we concentrate our study on the elliptical particles. In this article, we investigate numerically some metastable states including the quasiuniform state and

\footnotetext{
*Corresponding author. Tel.: + 886-2-33665183; fax: + 8862-23639984.

E-mail address: zhwei@phys.ntu.edu.tw (Z.-H. Wei).
}

several vortex states existing in an elliptical permalloy thin film. The energy densities of these metastable states as functions of particle's size are also discussed.

In our micromagnetic simulation we investigate permalloy elliptical particles by the integration of the Landau-Lifshitz-Gilbert equation [3]. The exchange energy, the magnetostatic energy, and the anisotropy energy are taken into account. The typical parameters of the permalloy material are as follows: The saturation magnetization $M_{\mathrm{s}}=800 \mathrm{emu} / \mathrm{cm}^{3}$, the exchange constant $C=2 A=2 \times 10^{-6} \mathrm{erg} / \mathrm{cm}$, and the uniaxial anisotropy constant $K_{1}=10^{3} \mathrm{erg} / \mathrm{cm}^{3}$. The elliptical particle is discretized into an array of small uniformly magnetized cubic cells with side length $5 \mathrm{~nm}$, which is small enough compared with the exchange length of the permalloy, $R_{0}=C^{1 / 2} / M_{\mathrm{S}}=17.7 \mathrm{~nm}$. The equilibrium spin configurations are obtained when each cell's largest angular variation between successive iterations is lower than $10^{-8}$.

Fig. 1 shows the simulation results of the typical spin configurations of the quasiuniform state, one-vortex state, two-vortex state, and three-vortex state in an elliptical permalloy thin film. The gray scale represents the magnetic pole density, which is derived from the divergence of the magnetization. They can be compared 

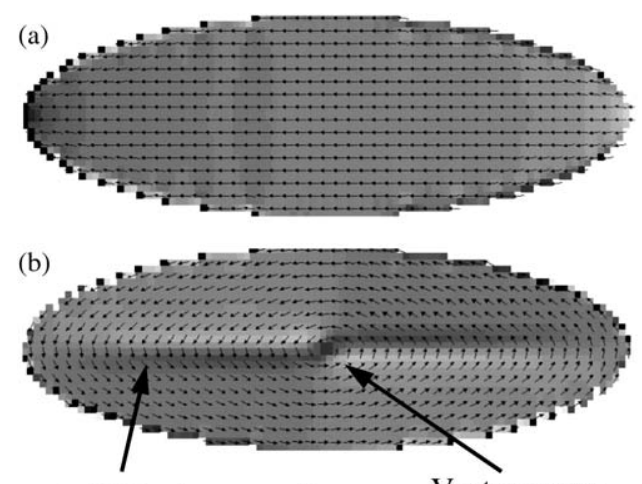

$180^{\circ}$ Neel type walls

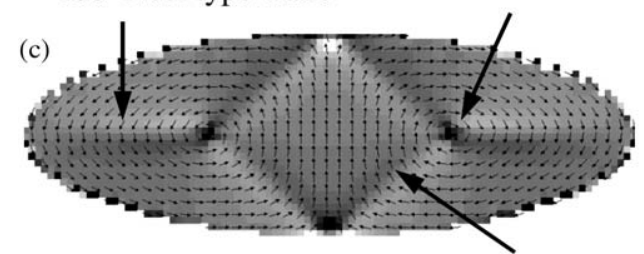

$90^{\circ}$ Neel type walls

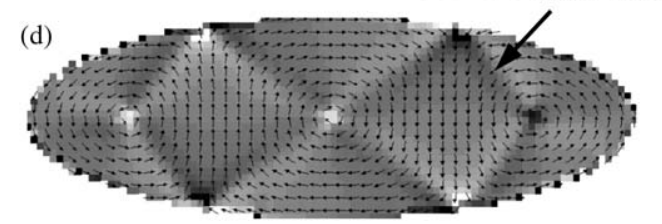

Fig. 1. Typical reduced spin configurations and magnetic pole densities: (a) quasiuniform state; (b) one-vortex state; (c) twovortex state; and (d) three-vortex state. The dimension of the elliptical element is $720 \mathrm{~nm}$ in long axis, $240 \mathrm{~nm}$ in short axis, and $30 \mathrm{~nm}$ in thickness.

with the images taken by magnetic force microscopy which measures the magnetic force gradient between the probe and the sample. The average magnetization of the quasiuniform state, which resembles the flower states for cubes and cylinders [4], is around $0.97 M_{\mathrm{s}}$ to $0.99 M_{\mathrm{s}}$. In contrast to the quasiuniform state, the average magnetization of the vortex states is almost negligible. The spin configurations of the vortex states consist of several vortices which have opposite sense of rotation with their neighbors. The magnetization of the vortex, except at the core, lies in-plane everywhere. The core, which extends to the order of the exchange length, locates at the vortex center and its magnetization is out-of-plane. In every vortex state shown in Fig. 1 the black/white region in the vortex core corresponds to the up/down magnetization. In fact, the energies of the vortex states are not influenced by the up or down direction of the core, and neighboring cores can independently have the same or opposite magnetizations. From Fig. 1 it can be easily observed that $180^{\circ}$ Neel type domain walls exist along the long axis at the one-vortex state. Also, in the

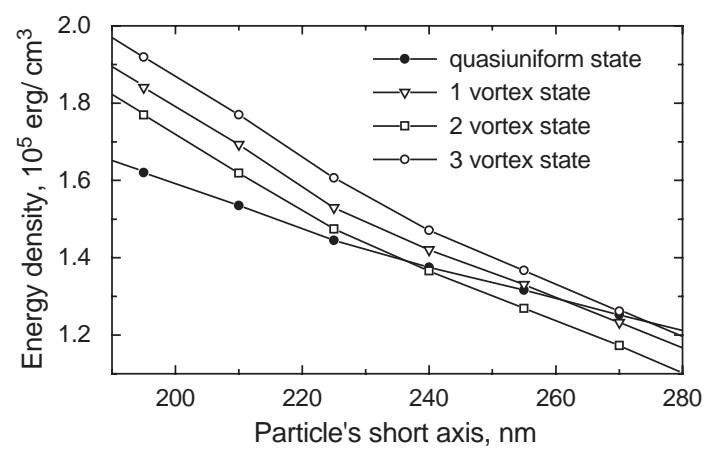

Fig. 2. Energy densities vs. particle's short axis in permalloy elliptical thin films with aspect ratio 3 .

two-vortex and three-vortex states two neighboring vortices form a rhombic domain, with nearly uniform magnetization directed parallel to the particle's short axis, surrounded by four $90^{\circ}$ Neel type domain walls. Deducing from the above, $\mathrm{N}$-vortex states can form $\mathrm{N}-1$ rhombic domains and the magnetizations of neighboring rhombic domains are in opposite directions.

Fig. 2 shows the typical simulation results carried out to obtain 'single-domain' size for elliptical element with aspect ratio 3 . The single-domain boundary is the critical size that the energy of the quasiuniform state equals those of the vortex states. Below the critical size the energy of the quasiuniform state is the lowest while above this size one of these vortex states has the lowest energy. When the particle size is smaller than $237 \mathrm{~nm}$ the energy of the quasiuniform state is the lowest while afterwards its energy gradually exceeds the energies of these vortex states. Besides, in thinner elements the magnetostatic energy decreases for the quasiuniform state while it increases for vortex states, so the critical size of thinner elements becomes larger. The energy densities of these vortex states decrease faster than that of the quasiuniform state as the in-plane size increases. Besides, the energies of these vortex states are very close to each other, and which state has the lowest energy depends on the size and aspect ratio of the particle.

This work was supported partly by the Republic of China National Science Council Grant No. NSC 922112-M-002-050 and Technology Development Program for AcademiaGrant No. 92-EC-17-A-08-S1-0006.

\section{References}

[1] L. Thomas, et al., Appl. Phys. Lett. 76 (2000) 766.

[2] W. Rave, et al., IEEE Trans. Magn. 36 (2000) 3886.

[3] M.E. Schabes, J. Magn. Magn. Mater. 95 (1991) 249.

[4] N.A. Usov, J. Magn. Magn. Mater. 130 (1994) 275. 